\title{
Joint User Selection and Multi-Mode Scheduling in Multicell MIMO Cellular Networks
}

\author{
Murtadha Al-Saedy, Mohammed Al-Imari, Mohammed Al-Shuraifi, and Hamed Al-Raweshidy
}

\begin{abstract}
This paper considers multicell scheduling for coordinated MIMO cellular networks. In a multicell scenario, intercell interference, which is the major challenge degrading system performance, can be suppressed via multicell coordination. However, when the number of users is larger than that can be served, user/multi-mode selection is necessary, and can significantly enhance the performance in such interference-limited environment. Nevertheless, user selection in a multicell scenario incurs high computational complexity and overhead signalling. To this end, in this paper, low complexity greedy algorithms are proposed for user selection. The algorithms are based on the concept of angle between subspaces and intersection of null spaces, and work in partially-distributed fashion with limited information exchange. By these algorithms, channel feedback can be greatly reduced with reasonable computational complexity. In addition, multimode scheduling schemes are proposed for this multicell set up, where the spatial streams are adaptively selected. The proposed algorithms are analysed and compared with the optimal solution obtained by exhaustive search. Simulation results show that the algorithms achieve most of the throughput achievable by the optimal solution.
\end{abstract}

Index Terms-Multicell coordination, multi-mode user selection, block diagonalization.

\section{INTRODUCTION}

Future wireless communication systems are expected to meet the explosive demand for high data rate applications [1]. Due to cost and scarceness of spectrum resources, wireless systems must be highly spectrally efficient [2]. Hence, the research has been growingly motivated towards increasing spectral efficiency. Various strategies and techniques have been proposed. Examples of such strategies are aggressive frequency reuse and dense deployment that have been thought as effective ways for exploiting the spectrum more efficiently. However, these strategies give rise to inter-cell interference which may severely degrade system performance [2], [3]. To come up with effective solutions for the inter-cell interference problem, different interference management strategies are proposed in this context such as interference avoidance, interference mitigation, and interference cancellation techniques [3].

Multi-user multiple-input multiple-output (MU-MIMO) has the potential of increasing system capacity by exploiting extra degrees of freedom offered by space domain. Multiple users can be served simultaneously on the same frequency band through spatial multiplexing and precoding techniques. The

M. Al-Saedy, M. Al-Shuraifi and H. Al-Raweshidy are with the Wireless Networks and Communications Centre, Brunel University, Uxbridge, UB8 3PH UK (e-mail: \{murtadha.al-saedy, mohammed.al-shuraifi, hamed.alraweshidy\}@brunel.ac.uk).

M. Al-Imari is with Samsung R\&D Institute UK, Staines-upon-Thames, Surrey, TW18 4QE, UK (e-mail: m.al-imari@samsung.com). channel can be decomposed into multiple parallel spatial subchannels to transmit multiple spatial data streams [4]. For optimal power allocation, water-filling strategy can be employed to allocate the power on these sub-channels [5]. Precoding techniques range from the one that has very high complexity such as dirty paper coding to the one with low complexity such as zero forcing (ZF) and block diagonalization (BD) [5]-[7]. The latter techniques are suitable for practical use and can asymptotically approach optimal performance when number of users gets very large [6].

The number of users that can be served by MU-MIMO is constrained by the transmit antennas. Hence, user selection needs to be implemented to choose the best spatially compatible users (their channels are uncorrelated) so that inter-user interference can be efficiently eliminated. Various algorithms are developed for user selection [6], [8]-[14]. For ZF, greedy user selection algorithms based on null-space successive projection and capacity-based metric are proposed in [6] and [8], respectively. For BD, capacity-based and Frobenius-based greedy algorithms are developed in [9]. However, these algorithms incur high complexity due to frequently using singular value decomposition (SVD) and water-filling. To overcome this limitation and avoid the unreliability caused by dealing with large concatenated matrix, a novel algorithm to iteratively select users is introduced in [10]. The algorithm is based on the idea of null spaces intersection where the pre-coder matrix of each user is obtained iteratively and sequentially to eliminate the inter-user interference and thereby incorporating it in user selection. Strategies based on angle between subspaces are proposed in [11]-[13]. This approach accounts for the spatial compatibility between users and relatively incurs less computational complexity. The authors of [13] utilise the iterative procedure in [10] to introduce an algorithm for user selection based on principal angle between subspaces. The idea of principal angle is further utilised with a different selection criteria based on capacity bound in [12]. These works assume that users utilise all their receive antennas. However, when the user has more antennas than can be served, a set of user antennas must be selected for reception. Antenna selection combined with user selection is addressed in [14], [15]. In [14], a simplified scheduling for antennas and user selection is proposed for MU-MIMO systems. While in [15], an adaptive strategy (multi-mode selection) for allocating varying number of spatial streams among users is developed employing capacity-based metric. More specifically, antennas and user selection are jointly performed so that an optimal subset of users and receive antennas are selected to maximise the sum capacity thereby it can better exploit multi-user diversity. 
In the aforementioned works, the focus has mainly been on single-cell scenarios and, therefore, losing practicality where the interference can not be ignored. Recently, to address intercell interference problem, MIMO technique is utilized on multicell system level in what has been known as multicell MIMO [16]. Two levels of multicell MIMO have been studied in the literature: coordinated MIMO and network MIMO [16]. In the first scheme, a cluster of base-stations (BS)s coordinate their beamforming transmission such that the interference is cancelled at users served by neighbouring cells [17]-[19]. In this strategy, global channel state information (CSI) of users in neighbouring cells must be exchanged among BSs. Whereas, in the later, a cluster of BSs act as a one giant BS jointly transmitting to their users so that the system can be viewed as a MU-MIMO [20]-[22]. In this case, data as well as CSI must be exchanged among the cells. Obviously network MIMO comes at the price of high cost in terms of control and data signals exchange [16].

Unlike the aforementioned works, in this paper, the problem of user scheduling in multicell setting is addressed. Assuming the number of users in each cell is larger than that can be served, the problem we consider here is how to select users in the cells with spatially separated channels such that the sumrate of the system is maximised. Furthermore, in addition to user selection, we also consider multi-mode selection problem where users may not all have the same number of spatial streams, thereby user selection is implemented across spatial modes. In both cases, solving multicell scheduling problem brings about high complexity in terms of computations and overhead signalling. To overcome these limitations, a distributed multicell scheduling algorithm is proposed consisting of two stages: multicell user scheduling stage and precoding stage.

In the multicell user scheduling stage, the algorithm works in a sequential manner such that in each cell, a BS selects its users based on CSIs of already selected users interference channels in other cells. In the second stage, having selected the sets of users by all BSs, precoding matrices are designed for the selected users in each cell. In both stages, each BS works separately from other BSs, thus no centralised action is required. Furthermore, two algorithms are proposed for the scheduling stage. In the first algorithm, named as distributed sequential user scheduling (DSUS), each BS schedules its set of users in its turn, where in each turn, users are selected such that the space spanned by users channels lies almost in the null space of interference channels of already selected users in the previous cells. The reason for doing so is to suppress the interference more efficiently. The process continues until all BSs select their users. In the other algorithm, named as distributed circular user scheduling (DCUS), users are iteratively scheduled across cells. Unlike DSUS algorithm, in DCUS, only one user is selected by each cell in each turn. Having all BSs selected one user per each, in the next round the process is repeated for selecting one user by each BS. In each user selection, a BS takes CSI of the so far selected users so that it can select another user in the null space of all users interference channels.

In the first algorithm, the cells have noticeable disparity in the sum-rate, where the last cell in selection order has higher sum-rate among the others. While, in the second algorithm, better fairness in terms of sum-rate among cells can be achieved compared to the first algorithm. This point will be further illustrated and justified in the subsequent sections.

In the procedure of multicell user selection described above, the order of BS sequence can be dictated by the system through central unit. Furthermore, no need to exchange all CSI of all users among the cells but only of those selected users.

The main contributions of the paper are summarised as follows:

- Low complexity user scheduling scheme is proposed for multicell setting with MIMO coordination. The proposed algorithm works in a partially-distributed manner with limited information exchange and consists of two stages: multicell scheduling and precoding stage. Thus, the proposed algorithm can greatly reduce overhead signalling by allowing only selected users to feedback their CSI.

- Two variants of multicell scheduling are proposed. The first algorithm has less complexity but leads to a gap in system performance among cells. While the second algorithm yields better fairness in terms of system performance though with more signalling iteration among the cells.

- The algorithm is extended to multi-mode selection in addition to the user selection, whereby the spatial modes are adaptively selected in each cell.

The remainder of the paper is organised as follows. Section II describes the proposed system model and the concept of BD and principal angle. Section III presents the problem of multicell scheduling and introduces the proposed algorithms and its two variants and how the proposed algorithm is extended to multi-mode selection. In Section IV, fair scheduling is introduced for the proposed algorithm. Section V presents the simulation results of the proposed algorithm. Finally, conclusions are drawn in Section VI.

Notations: Bold and lower-case letters denote vectors while bold and upper-case letters denote matrices. $\mathbf{I}_{n \times n}$ denotes the identity matrix with $n \times n$ dimensions. The notations $(.)^{\mathrm{H}},\|\cdot\|$, and $\operatorname{tr}\{$.$\} denote conjugate transpose, norm of a vector, and$ trace of a matrix, respectively. $|\mathcal{A}|$ denotes the cardinality of set $\mathcal{A} . \mathcal{N}($.$) and \mathcal{R}($.$) denote the null space and row space$ of a matrix, respectively. $\mathbb{E}\{$.$\} is the expectation operator.$ Furthermore, null $(\mathbf{M})$ and $\operatorname{row}(\mathbf{M})$ denote the matrices whose columns form an orthonormal basis of $\mathcal{N}(\mathbf{M})$ and $\mathcal{R}(\mathbf{M})$, respectively.

\section{SYSTEM MODEL}

\section{A. Signal Model and Block Diagonalization}

Consider a downlink cellular network consisting of a set of $\mathcal{B}$ BSs, where $\mathcal{B}=\{1, \ldots, B\}$ as shown in Fig. 1 for the case of $B=3$. Each BS is equipped with $N_{t}$ antennas and serves users, each equipped with $N_{r}$ antennas. Suppose that the active set of users to be selected and served by BS $b$ is $\mathcal{K}_{b} \in \mathcal{U}_{b}$, where $\mathcal{U}_{b}$ is the set of all users in cell $b$, and denote $k_{b}$ as an index of a user served by BS $b$ such that 


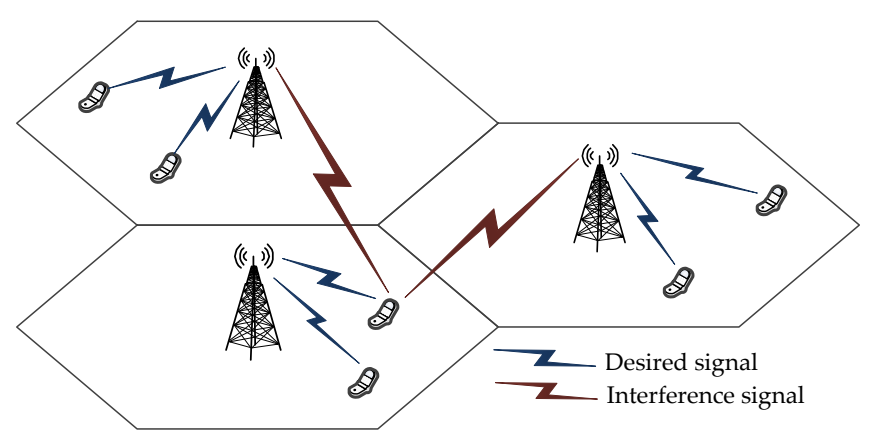

Fig. 1. Illustration of coordinated MIMO cellular network, each BS serves multiple users equipped with multiple antennas.

$k_{b} \in \mathcal{K}_{b}=\left\{1, \ldots,\left|\mathcal{K}_{b}\right|\right\}$. The downlink channel matrix from set of $\mathcal{B}$ BSs to user $k_{b}$ in cell $b$ is given by:

$$
\mathbf{H}_{k_{b}}=\left[\sqrt{\beta_{k_{b}}^{1}} \mathbf{H}_{k_{b}}^{(1)}, \sqrt{\beta_{k_{b}}^{2}} \mathbf{H}_{k_{b}}^{(2)}, \ldots, \sqrt{\beta_{k_{b}}^{B}} \mathbf{H}_{k_{b}}^{(B)}\right],
$$

where $\mathbf{H}_{k_{b}}^{(i)} \in \mathbb{C}^{N_{r} \times N_{t}}$ is the downlink channel matrix from BS $i$ to user $k_{b}$ located at BS $b$. Each component of $\mathbf{H}_{k_{b}}^{(i)}$ is an independent identically distributed complex Gaussian random variable with zero-mean and unit-variance. $\beta_{k_{b}}^{i}$ denotes the large scale channel fading (path-loss and shadowing) between $i$ th BS and $k_{b}$ user. The transmit data vector of user $k_{b}$ is given by $\mathbf{x}_{k_{b}} \in \mathbb{C}^{L_{k_{b}} \times 1}$, where $L_{k_{b}}$ denotes the number of spatial streams allocated for user $k_{b}$. The data vector $\mathbf{x}_{k_{b}}$ is multiplied by $N_{t} \times L_{k_{b}}$ precoding matrix $\mathbf{P}_{k_{b}}$ and the resultant signal is transmitted through $N_{t}$ antennas from BS $b$.

At the receiver side of user $k_{b}$, a post-processing matrix $\mathbf{W}_{k_{b}} \in \mathbb{C}^{L_{k_{b}} \times N_{r}}$ is employed to decode the spatial streams at the receiver. Hence, the received signal $\mathbf{y}_{k_{b}} \in \mathbb{C}^{L_{k_{b}} \times 1}$ after post-processing is given by:

$$
\begin{gathered}
\mathbf{y}_{k_{b}}=\underbrace{\mathbf{W}_{k_{b}}^{\mathrm{H}} \mathbf{H}_{k_{b}}^{(b)} \mathbf{P}_{k_{b}} \mathbf{x}_{k_{b}}}_{\text {desired signal }}+\underbrace{\mathbf{W}_{k_{b}}^{\mathrm{H}} \mathbf{H}_{k_{b}}^{(b)} \sum_{i_{b}=1, i_{b} \neq k_{b}}^{\left|\mathcal{K}_{b}\right|} \mathbf{P}_{i_{b}} \mathbf{x}_{i_{b}}}_{\text {intra-cell interference }} \\
+\underbrace{\mathbf{W}_{k_{b}}^{\mathrm{H}} \sum_{s=1, s \neq b}^{B} \mathbf{H}_{k_{b}}^{(s)} \sum_{n_{s}=1}^{\left|\mathcal{K}_{s}\right|} \mathbf{P}_{n_{s}} \mathbf{x}_{n_{s}}}_{\text {inter-cell interference }}+\mathbf{W}_{k_{b}}^{\mathrm{H}} \mathbf{n}_{k_{b}},
\end{gathered}
$$

where $\mathbf{n}$ is the additive white Gaussian noise (AWGN) such that $\mathbb{E}\left\{\mathbf{n}_{k_{b}} \mathbf{n}_{k_{b}}^{\mathrm{H}}\right\}=\sigma^{2} \mathbf{I}$. The second term is the intra-cell interference that stems from transmission to the users served by the same BS. The third term is the inter-cell interference that comes from other BSs. For the sake of complete exposition, we will describe the whole process of interference cancellation. To cancel the intra-cell interference, the following should be satisfied [7]:

$$
\mathbf{H}_{k_{b}}^{(b)} \mathbf{P}_{i_{b}}=0, \quad \forall k_{b} \neq i_{b}, \forall k_{b}, i_{b} \in \mathcal{K}_{b} .
$$

On the other hand, to cancel the inter-cell interference, the following should also be satisfied:

$$
\mathbf{H}_{k_{b}}^{(s)} \mathbf{P}_{n_{s}}=0, \quad \forall k_{b} \in \mathcal{K}_{b}, n_{s} \in \mathcal{K}_{s}, b \neq s \in \mathcal{B} .
$$

To satisfy the condition in (3), i.e. cancelling the intra-cell interference, the precoding matrix $\mathbf{P}_{k_{b}}$ should lie in the null space of $\tilde{\mathbf{H}}_{k_{b}}^{b}$, which is defined as [7], [8]

$$
\tilde{\mathbf{H}}_{k_{b}}^{(b)}=\left[\mathbf{H}_{1}^{(b)^{\mathrm{H}}}, \ldots, \mathbf{H}_{k_{b}-1}^{(b)^{\mathrm{H}}}, \mathbf{H}_{k_{b}+1}^{(b)^{\mathrm{H}}}, \ldots, \mathbf{H}_{\left|\mathcal{K}_{b}\right|}^{(b]^{\mathrm{H}}}\right]^{\mathrm{H}} .
$$

Whereas, to cancel out the inter-cell interference, the precoding matrix $\mathbf{P}_{k_{b}}$ should lie in the null space of $\hat{\mathbf{H}}_{k_{b}}^{(b)}$ which is defined as:

$\hat{\mathbf{H}}_{k_{b}}^{(b)}=\left[\overline{\mathbf{H}}_{1}^{(b)^{\mathrm{H}}}, \ldots, \overline{\mathbf{H}}_{s-1}^{(b)^{\mathrm{H}}}, \overline{\mathbf{H}}_{s+1}^{(b)}, \ldots, \overline{\mathbf{H}}_{B}^{(b)^{\mathrm{H}}}\right]^{\mathrm{H}}, \quad \forall b \neq s \in \mathcal{B}$,

where we define $\overline{\mathbf{H}}_{s}^{(b)}$ as:

$$
\overline{\mathbf{H}}_{s}^{(b)}=\left[\mathbf{H}_{1}^{(b)^{\mathrm{H}}}, \ldots, \mathbf{H}_{\left|\mathcal{K}_{s}\right|}^{(b)^{\mathrm{H}}}\right]^{\mathrm{H}} .
$$

To obtain the precoders that satisfy the conditions in (3) and (4), each BS $b$ concatenates channel matrices of its own users along with interference channels to the users in other cells as $\widehat{\mathbf{H}}_{k_{b}}^{(b)}=\left[\tilde{\mathbf{H}}_{k_{b}}^{(b)}, \hat{\mathbf{H}}^{(b)^{\mathrm{H}}}\right]^{\mathrm{H}}$. Thus, to nullify both intercell and intra-cell interference using $\mathrm{BD}$, the pre-coder $\mathbf{P}_{k_{b}}$ must be constructed such that:

$$
\widehat{\mathbf{H}}_{k_{b}}^{(b)} \mathbf{P}_{k_{b}}=0, \quad \forall b \neq s \in \mathcal{B} .
$$

Let $\widehat{l}_{k_{b}}=\operatorname{Rank}\left(\widehat{\mathbf{H}}_{k_{b}}^{(b)}\right)$, the condition in (8) can only be satisfied when $\operatorname{Rank}\left(\widehat{\mathbf{H}}_{k_{b}}^{b)}\right)<N_{t}$. Let the singular value decomposition (SVD) of $\widehat{\mathbf{H}}_{k_{b}}^{(b)}$ as $\widehat{\mathbf{H}}_{k_{b}}^{(b)}=\widehat{\mathbf{U}}_{k_{b}}^{(b)} \widehat{\boldsymbol{\Lambda}}_{k_{b}}^{(b)}\left[\widehat{\mathbf{V}}_{k_{b}, 1}^{(b)} \widehat{\mathbf{V}}_{k_{b}, 0}^{(b)}\right]^{\mathrm{H}}$, where $\widehat{\Lambda}_{k_{b}}^{(b)}$ is the $\widehat{l}_{k_{b}} \times \widehat{l}_{k_{b}}$ diagonal matrix, i.e. $\widehat{\Lambda}_{k_{b}}^{(b)}=$ $\operatorname{diag}\left(\lambda_{1, k_{b}}, \ldots, \lambda_{\widehat{l}_{k_{b}}, k_{b}}\right), \widehat{\mathbf{V}}_{k_{b}, 1}^{(b)}$ contains the first $\widehat{l}_{k_{b}}$ right singular vectors, $\widehat{\mathbf{U}}_{k_{b}}^{(b)}$ is an orthogonal matrix and $\widehat{\mathbf{V}}_{k_{b}, 0}^{(b)}$ contains the last $N_{t}-\widehat{l}_{k_{b}}$ right singular vectors. Therefore, the columns of $\widehat{\mathbf{V}}_{k_{b}, 0}^{(b)}$ form the null space basis of $\widehat{\mathbf{H}}_{k_{b}}^{(b)}$. From $\widehat{\mathbf{V}}_{k_{b}, 0}^{(b)}$, the precoding matrix $\mathbf{P}_{k_{b}}$ can be constructed such that both intracell and inter-cell interference can be eliminated [7]-[9].

The precoding matrix $\mathbf{P}_{k_{b}}$ can be decomposed into three matrices $\mathbf{Z}_{k_{b}}^{1}, \mathbf{Z}_{k_{b}}^{2}$, and $\mathbf{B}_{k_{b}}$, i.e. $\mathbf{P}_{k_{b}}=\mathbf{Z}_{k_{b}}^{1} \mathbf{Z}_{k_{b}}^{2} \mathbf{B}_{k_{b}}$. where $\mathbf{Z}_{k_{b}}^{1}$ is designed to eliminate intra-cell interference, therefore it should lie in $\mathcal{N}\left(\tilde{\mathbf{H}}_{k_{b}}^{(b)}\right)$. While $\mathbf{Z}_{k_{b}}^{2}$ is designed to eliminate inter-cell interference, thus it should lie in $\mathcal{N}\left(\hat{\mathbf{H}}_{k_{b}}^{(b)}\right)$. $\mathbf{B}_{k_{b}}$ is the matrix holding singular vectors that maximise data rate. Let $\ddot{\mathbf{H}}_{k_{b}}^{(b)}$ denote the effective channel matrix for user $k_{b}$, i.e. $\ddot{\mathbf{H}}_{k_{b}}^{(b)}=\mathbf{H}_{k_{b}}^{(b)} \mathbf{Z}_{k_{b}}^{1} \mathbf{Z}_{k_{b}}^{2}$. To obtain $\mathbf{B}_{k_{b}}$ that maximises data rate, we find SVD of $\ddot{\mathbf{H}}_{k_{b}}^{(b)}$ as:

$$
\ddot{\mathbf{H}}_{k_{b}}^{(b)}=\ddot{\mathbf{U}}_{k_{b}}^{(b)}\left[\ddot{\boldsymbol{\Lambda}}_{k_{b}}^{(b)}, \mathbf{0}\right]\left[\ddot{\mathbf{V}}_{k_{b}, 1}^{(b)} \ddot{\mathbf{V}}_{k_{b}, 0}^{(b)}\right]^{\mathrm{H}},
$$

where $\ddot{\mathbf{V}}_{k_{b}, 1}^{(b)}$ contains the first right singular vectors, the columns of which correspond to the orthonormal basis of $\mathcal{R}\left(\ddot{\mathbf{H}}_{k_{b}}^{(b)}\right)$. Thus, by setting $\mathbf{B}_{k_{b}}=\ddot{\mathbf{V}}_{k_{b}, 1}^{(b)}$, the data rate is maximised. The method to design precoding matrices mentioned above utilises SVD to find the null space for each user. The issue with this method is that it incurs costly computations. Motivated by the iterative method introduced in [10], the null space matrices can be obtained iteratively by utilising the idea of null space intersection [23]. More specifically, consider the matrix that nullifies the intra-cell interference of user 1 , i.e. $\mathbf{Z}_{1}^{1}$. Let $\mathbf{Z}_{1}^{1^{(i)}}$ be precoding matrix $\mathbf{Z}_{1}^{1}$ after $i$ th iteration, $\mathbf{Z}_{1}^{1^{(i)}}$ 


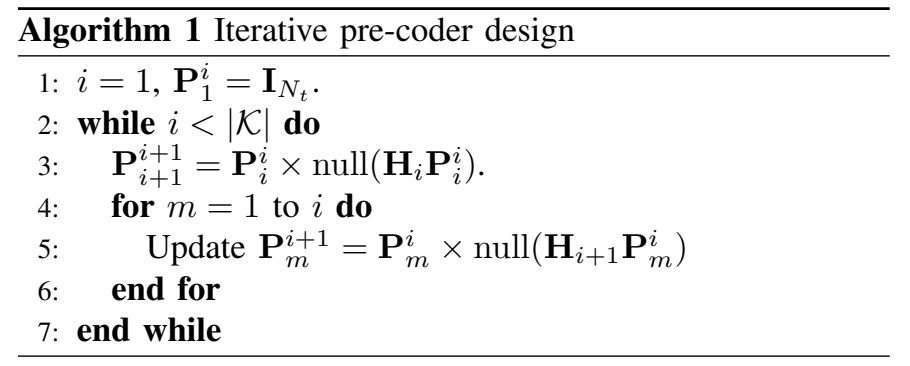

should satisfy that $\mathbf{H}_{k_{b}}^{(b)} \mathbf{Z}_{1}^{1^{(i)}}=0$ for all $1<k_{b} \leq i$. Then, $\mathbf{Z}_{1}^{1^{(i+1)}}$ can be found as:

$$
\mathbf{Z}_{1}^{1^{(i+1)}}=\mathbf{Z}_{1}^{1^{(i)}} \mathbf{G}
$$

where the columns of $\mathbf{G}$ lie in the null space of $\mathbf{H}_{i+1}^{(b)} \mathbf{Z}_{1}^{1^{(i)}}$, i.e. $\mathcal{N}\left(\mathbf{H}_{i+1}^{(b)} \mathbf{Z}_{1}^{1^{(i)}}\right)$. In the same manner, the precoding matrices of other users can be obtained iteratively. In each iteration, the number of columns of $\mathbf{Z}_{1}^{1^{(i)}}$ is reduced by $N_{r}$. Algorithm 1 summarises the aforementioned procedure [10]. The BS index is removed from the notations in Algorithm 1 for simplicity.

\section{B. Principal Angle Between Subspaces}

To utilise the iterative procedure for user selection, we use an approach based on angle between subspaces of users channels. Here, we review the concepts of principal angle and geometrical angle utilised in this paper. Assume that $\mathcal{U}$, $\mathcal{V} \subset \mathbb{C}^{n}$ are two non-zero subspaces, the principal angles $\delta_{i} \in[0, \pi / 2], i=1, \ldots, m$, between $\mathcal{U}$ and $\mathcal{V}$ are recursively defined such that [24]:

$$
\begin{aligned}
& \cos \left(\delta_{i}\right)=\max _{\mathbf{u} \in \mathcal{U}, \mathbf{v} \in \mathcal{V}} \mathbf{u}^{\mathrm{H}} \mathbf{v} \\
&= \\
& \mathbf{u}_{i}^{\mathrm{H}} \mathbf{v}_{i}, \\
& \text { subject to, }\|\mathbf{u}\|=\|\mathbf{v}\|=1,
\end{aligned}
$$

where $m=\min \{\operatorname{dim}(\mathcal{U}), \operatorname{dim}(\mathcal{V})\}, \mathbf{u}$ and $\mathbf{v}$ are the vectors that form the $i$ th principal angle. $\cos \left(\delta_{i}\right)$ can be defined in terms of the eigenvalues as follows [24], [25]:

$$
\cos ^{2}\left(\delta_{i}\right)=\lambda_{i}, \quad i=1, \ldots, m,
$$

where $\lambda_{i}$ are the eigenvalues of $\widehat{\mathbf{P}}_{1}^{\mathrm{H}} \widehat{\mathbf{P}}_{2} \widehat{\mathbf{P}}_{2}^{\mathrm{H}} \widehat{\mathbf{P}}_{1}$, with $\widehat{\mathbf{P}}_{1}$ and $\widehat{\mathbf{P}}_{2}$ the orthonormal basis matrices of subspaces $\mathcal{U}$ and $\mathcal{V}$, respectively.

Principal angles can be used as an indication of the degree of spatial correlation. That is, the larger the principal angle is, the more uncorrelated subspaces are. Thus, principal angles can be utilised to measure the orthogonality between users channels. To fully characterise spatial correlation between two subspaces, it is beneficial to consider the concept of geometrical angle. In the following, the definition of geometric angle and its relation to singular values will be given.

Geometrical Angle: For the given subspace $\mathcal{U}$ and $\mathcal{V}$, the geometrical angle, i.e. the angle $\Theta=\measuredangle(\mathcal{U}, \mathcal{V})$ between the two subspaces, is defined as [24], [25].

$$
\begin{aligned}
\cos ^{2}(\Theta) & =\prod_{i=1}^{m} \cos ^{2}\left(\delta_{i}\right) \\
& =\operatorname{det}\left(\widehat{\mathbf{P}}_{1}^{\mathrm{H}} \widehat{\mathbf{P}}_{2} \widehat{\mathbf{P}}_{2}^{\mathrm{H}} \widehat{\mathbf{P}}_{1}\right) .
\end{aligned}
$$

$\cos ^{2}(\Theta)$ represents the ratio between the volumes of the parallelepiped spanned by the projection of the basis vectors of the lower dimension subspace on the higher dimension subspace and the one spanned by the basis vectors of the lower dimension subspace [26].

Based on both principal angle and the previously described iterative procedure, the authors of [13] propose low complexity algorithm for user selection in single cell scenario. In the next section, the algorithm is subtly modified and utilised in the proposed multicell scheduling.

\section{Multicell User Scheduling Algorithms}

In this section, we define the problem of multicell scheduling that maximise sum-rate of the system. In order to make the analysis more consistent, we start first with single cell scheduling and then, subsequently, proceed to define multicell scheduling problem. The reason behind this will be clarified in the next discussions.

\section{A. Single Cell scheduling}

In a single cell scenario, inter-cell interference is not considered. Hence, as described previously, scheduling users can be either implemented with fixed number of spatial modes being allocated for each user, which corresponds to the conventional $\mathrm{BD}$, or with varying number of spatial modes per each user, which corresponds to the multi-mode selection. In the later case, the selected users may have different number of spatial modes. One way to implement multi-mode scheduling is by the way of antenna selection. In antenna selection, the scheduler should select the best set of antennas that maximises the sum-rate in the system. Multi-mode scheduling allows more flexibility in allocating the spatial modes and can substantially improve data rate by exploiting multi-mode diversity [15]. Even though throughput of a user can be decreased, nevertheless, it frees up the resources so that they can be used by other users with better spatial channel separation. Hence, in general, the sum-rate of maximization with conventional $\mathrm{BD} /$ multi-mode scheme in single cell is written as

$$
\begin{aligned}
& R_{b}=\max _{\mathcal{K}_{b} \in \mathcal{U}_{b}, L_{k_{b}}, \mathbf{P}_{k_{b}}, \mathbf{Q}_{k_{b}}} \sum_{k_{b} \in \mathcal{K}_{b}} \log _{2} \operatorname{det}(\mathbf{I}+ \\
&\left.\frac{1}{\sigma^{2}} \mathbf{H}_{k_{b}}^{(b)} \mathbf{P}_{k_{b}} \mathbf{Q}_{k_{b}} \mathbf{P}_{k_{b}}^{\mathrm{H}} \mathbf{H}_{k_{b}}^{(b) \mathrm{H}}\right),
\end{aligned}
$$

subject to

$$
\begin{gathered}
\sum_{k_{b}}^{\left|\mathcal{K}_{b}\right|} L_{b_{k}} \leq N_{t}, \\
\sum_{k_{b}=1}^{\left|\mathcal{K}_{b}\right|} \operatorname{tr}\left(\mathbf{Q}_{k_{b}}\right) \leq P .
\end{gathered}
$$

For conventional BD, $L_{b_{k}}$ is fixed, i.e. $L_{b_{k}} \leq N_{r}$, and for multi-mode selection it can take the values $0 \leq L_{b_{k}} \leq N_{r}$ for each user at each scheduling instant. $\mathbf{Q}_{k_{b}}$ and $P$ are the transmit covariance matrix of size $L_{k_{b}}$ and the total transmit power allowed for each BS, respectively. Note that the covariance matrix $\mathbf{Q}_{k_{b}}$ can be determined by water-filling 
over non-zero eigenmodes of the effective channel. However, it is well known that equal power allocation performs as good as water-filling in high signal to noise ratio (SNR) regime. Thus, we will consider equal power allocation for the rest of analysis, then we have $\mathbf{Q}_{k_{b}}=\left(P / \sum_{i}^{\left|\mathcal{K}_{b}\right|} L_{b_{k}}\right) \mathbf{I}_{L_{b_{k}} \times L_{b_{k}}}$.

In general, only exhaustive search can solve this problem by searching over all possible sets of users/spatial modes, which is highly computationally costly. Therefore, we propose two suboptimal low complexity algorithms for both conventional $\mathrm{BD}$ and multi-mode user scheduling schemes. Although they are for single cell scheduling, the proposed algorithms will take into account the interference from other cells. Thus, they will be utilized later for our proposed iterative multicell scheduling algorithms. In the following discussion, we briefly explain the algorithms and how they work.

a) Scheduling with fixed number of spatial modes (conventional $B D$ ): when the transmission from the BS to each user utilises all available receive antennas at the users, no antenna (or multi-mode) selection is needed and $L_{b_{k}}=N_{r}$. Hence, the channel matrix between BS $b$ and the user $k_{b}$ is $\mathbf{H}_{k_{b}}^{(b)} \in \mathbb{C}^{N_{r} \times N_{t}}$. Accordingly, scheduling can be implemented to choose the best highly uncorrelated users channels.

Here, we propose a greedy user selection algorithm based on principal angle concept [13]. Consider a cell $b$, at the initialisation step, the algorithm computes the null space of the interference channels of users in other cells, denoted as $\mathbf{N}$. The null space $(\mathbf{N})$ is used to compute $\mu_{k_{b}}^{(1)}$ for all users in the cell, where $\mu_{k_{b}}$ represents the product of eigenvalues of the effective channel for user $k_{b}$. Then, the user with the maximum $\mu_{k_{b}}$ is selected, and a null space matrix $\mathbf{W}$ is initialised based on the selected user and the matrix $\mathbf{N}$, according to step (5). Note that $\mathcal{W}_{b}$ is the sets of unselected users. At the $i$ th iteration, the algorithm updates users' metrics and selects the $i$ th user satisfying (27). After the user is selected, $\mathcal{K}_{b}, \mathcal{W}_{b}$ and $\mathrm{W}$ are updated accordingly. The conventional BD scheduling algorithm is detailed in Algorithm 2.

It is worth mentioning that, comparing to conventional single cell algorithms, our algorithm considers the null space (N) in calculating the users' metrics $\left(\mu_{k_{b}}\right)$ and the updated null space matrix $\mathbf{W}$, which will subsequently affect the users' selection. The inclusion of $\mathbf{N}$ yields further intersection with null space of other users interference channels.

b) Scheduling with multi-mode selection: when the number of spatial modes are adaptively allocated for each user, both user and spatial mode selection need to be implemented. In Algorithm 3, we modify Algorithm 2 to incorporate spatial multi-mode selection. The algorithm works by checking the best antenna of a user that has maximum space angle with the subspace spanned by already selected antennas. Accordingly, the channel matrix between BS $b$ and antenna $l$ at user $k_{b}$ is represented by $\mathbf{h}_{k_{b}, l}^{(b)} \in \mathbb{C}^{1 \times N_{t}}$. Denote $\mathcal{A}_{k_{b}}$ and $\mathcal{S}_{k_{b}}$ as sets of remaining antennas and selected antennas for user $k_{b}$ in a cell $b$, respectively. $\mathcal{W}_{b}$ is the set of users in cell $b$ that have some antennas still unselected. While $\mathcal{K}_{b}$ is the set of users that have antennas selected.

The Algorithms 2 and 3 will be used for multicell schedul-

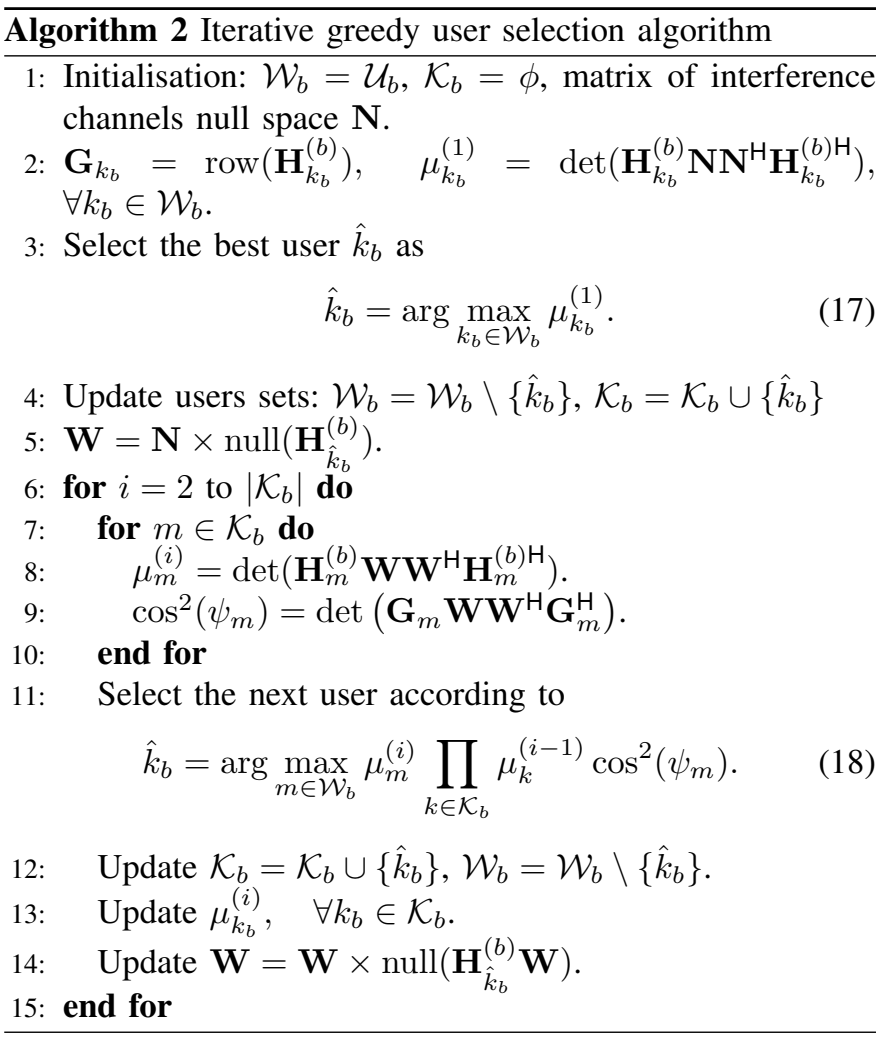

ing algorithms presented in the next section. They can be used by each cell to select its own users/spatial modes such that the selected users channel directions have maximum subspace angle with interference channels of the previous users cells. Further elaboration will be made to describe this process in the next section.

\section{B. Multicell Scheduling Problem Formulation}

When considering multicell scenario, the problem of finding the best users set for each cell $\mathcal{K}_{b} \in \mathcal{U}_{b}, \forall b \in \mathcal{B}$, gets coupled with precoding design due to inter-cell interference. Hence, scheduling users across multiple cells becomes more complicated, since we have to jointly schedule users/spatial modes sets across all cells. Thus, multicell scheduling that maximises the sum-rate for $B$ cells is mathematically expressed as

$$
\begin{array}{r}
R_{\text {sum }}=\max _{\mathcal{K}_{b} \in \mathcal{U}_{b}, L_{k_{b}}, \mathbf{P}_{k_{b}}, \mathbf{Q}_{k_{b}}} \sum_{b=1}^{B} \sum_{k_{b} \in \mathcal{K}_{b}} \log _{2}(\operatorname{det}(\mathbf{I}+ \\
\left.\left.\frac{\mathbf{H}_{k_{b}}^{(b)} \mathbf{P}_{k_{b}} \mathbf{Q}_{k_{b}} \mathbf{P}_{k_{b}}^{\mathrm{H}} \mathbf{H}_{k_{b}}^{(b) \mathrm{H}}}{\sum_{s \neq b} \mathbf{H}_{k_{b}}^{(s)} \mathbf{P}_{k_{s}} \mathbf{Q}_{k_{s}} \mathbf{P}_{k_{s}}^{\mathrm{H}} \mathbf{H}_{k_{b}}^{(s) \mathrm{H}}+\sigma^{2}}\right)\right),
\end{array}
$$

subject to

$$
\begin{gathered}
\sum_{k_{b}}^{\left|\mathcal{K}_{b}\right|} L_{b_{k}} \leq N_{t}-B\left|\mathcal{K}_{b}\right|, \quad \forall b \in \mathcal{B}, \\
0 \leq L_{b_{k}} \leq N_{r}, \\
\sum_{k_{b}=1}^{\left|\mathcal{K}_{b}\right|} \operatorname{tr}\left(\mathbf{Q}_{k_{b}}\right) \leq P, \quad \forall b \in \mathcal{B},
\end{gathered}
$$




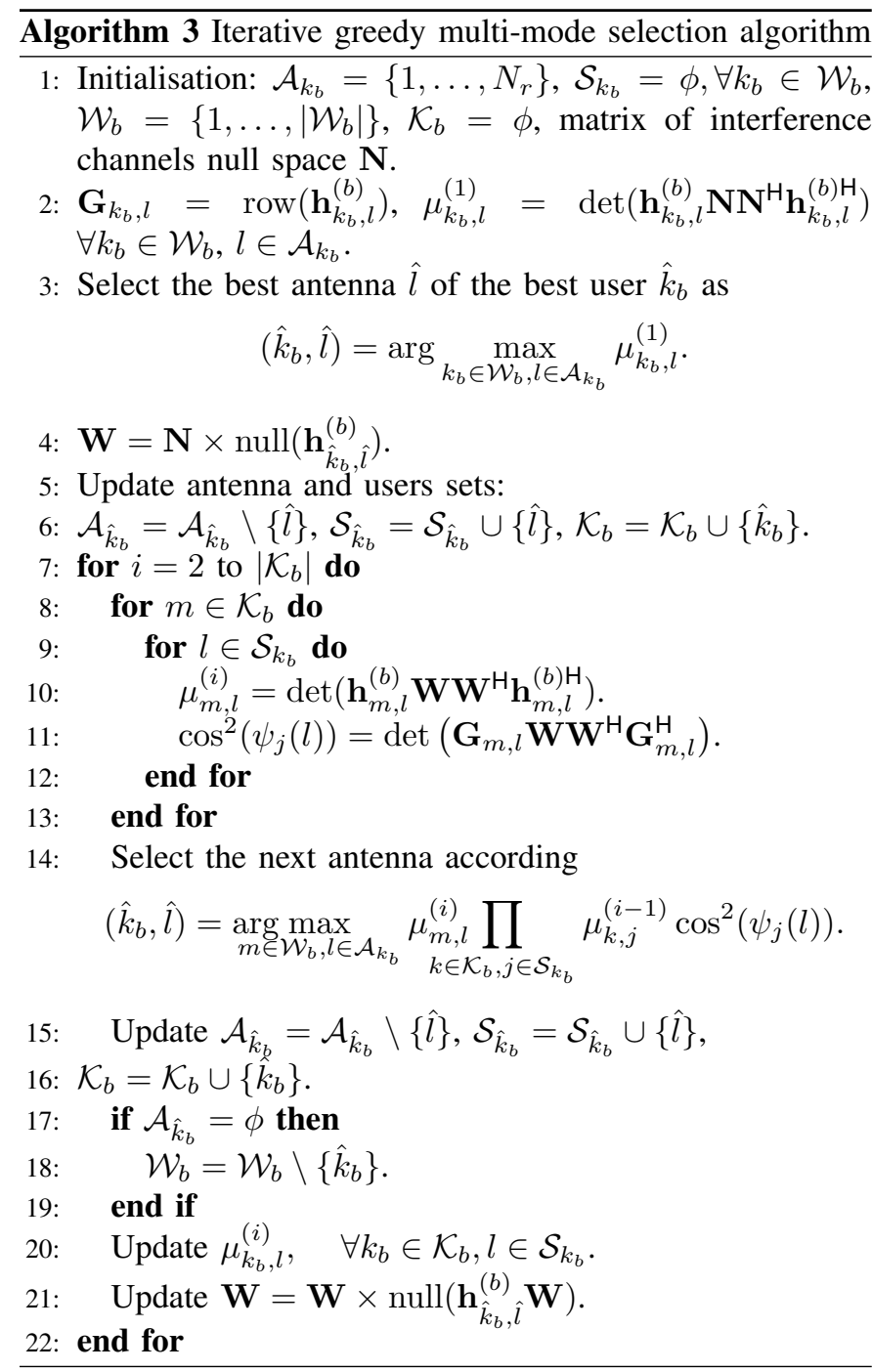

$$
\begin{gathered}
\mathbf{H}_{k_{b}}^{(b)} \mathbf{P}_{i_{b}}=0, \quad \forall k_{b} \neq i_{b}, \\
\mathbf{H}_{k_{b}}^{(s)} \mathbf{P}_{n_{s}}=0, \quad \forall k_{b} \in \mathcal{K}_{b}, n_{s} \in \mathcal{K}_{s}, b \neq s \in \mathcal{B} .
\end{gathered}
$$

Evidently, solving the problem (19) in realistic systems faces three major challenges summarised as follow:

- Inter-cell interference: the first challenge is the inter-cell interference coming from neighbouring BSs. To overcome this problem, BSs can coordinate their beamforming or precoding matrices so that the interference is eliminated at all users. To better utilise coordinated MIMO technique, the set of users in the cells should be selected such that each set of users in a cell have maximum spatial separation with interference channels of users in the other cells.

- Computational complexity: the second challenge is how to find an optimal set of users in each cell such that the intercell interference is perfectly eliminated, while maximising the sum-rate of all cells. To clarify this point, without loss of generality consider two cells; $b_{1}$ and $b_{2}$ serving users $k_{b_{1}}$ and $k_{b_{2}}$, respectively. For the $b_{1}$ to perfectly eliminate the interference to user $k_{b_{2}}$ while serving its user $k_{b_{1}}$, the channel direction of $b_{1}$ from its served user $k_{b_{1}}$, i.e. $\mathbf{H}_{k_{b_{1}}}^{b_{1}}$ and its interference channel to the user $k_{b_{2}}$, i.e. $\mathbf{H}_{k_{b_{2}}}^{b_{1}}$ must have per- fect orthogonality. When there are more users than that can be served, only exhaustive search can find the optimal solution for this problem by searching over all possible subsets of users and spatial modes given by $\sum_{i=1}^{\left\lceil B N_{t} / N_{r}\right\rceil} C\left(B\left|\mathcal{U}_{b}\right|, i\right)$ and $\sum_{i=1}^{\left\lceil B N_{t}\right\rceil} C\left(B\left|\mathcal{U}_{b}\right| N_{r}, i\right)$, respectively, where $C(n, k)$ denotes the number of $k$-combinations from a given set of $n$ elements. This induces complexity given by

$$
\begin{aligned}
\varphi_{u s} & \approx \mathcal{O}\left(\left|\mathcal{K}_{b}\right|\left(B N_{t}\right)^{3} C\left(B\left|\mathcal{U}_{b}\right|,\left|\mathcal{K}_{b}\right|\right)\right), \\
\varphi_{m m} & \approx \mathcal{O}\left(\frac{\left(B N_{t}\right)^{5}}{N_{r}} C\left(B\left|\mathcal{U}_{b}\right| N_{r}, B N_{t}\right)\right) .
\end{aligned}
$$

It is clear from (25) and (26) that the complexity becomes prohibitive as the total number of users in the cell $\left|\mathcal{U}_{b}\right|$ grows large. It can be observed that the problem becomes even harder due to the coupling in user selection among cells. Consequently, the computational complexity of exhaustive search becomes even higher than that of a single cell user selection.

- CSI feedback cost: assuming perfect CSI feedback and centralised processing for selecting users across cells, the BSs should exchange the global CSI of all users at each scheduling period, which puts too much burden to be permitted on the limited-capacity backhaul links. Moreover, when the number of users in each cells gets larger, CSI cost increases accordingly. This holds true even when no exhaustive search but centralised processing is implemented.

Motivated by the previous discussion, a suboptimal low complexity algorithm is necessary from the practical point of view for user selection in multicell setting to avoid complexity involved in (19), while requiring less CSI sharing among cells. To decouple the joint problem (19) into two sub-problems, we propose two stages procedure; scheduling stage and precoding stage. In the scheduling stage, BSs progressively and sequentially select their users in their coverage region such that each BS selects its users separately from all other cells. At each step, each BS, say $b$, takes a limited amount of information from the previous BSs ( 1 to $b-1$ ), and based on which it selects its users. Two types of algorithms are proposed to implement this procedure; DSUS and DCUS. In the following discussion, each algorithm will be described thoroughly. Without loss of generality, let us assume fixed spatial stream case, i.e. conventional BD, when discussing the multicell algorithms; nevertheless, the algorithms are also applicable to multi-mode case.

a) DSUS algorithm: in this algorithm, multicell scheduling is implemented in sequential distributed manner. At the initialization, the first BS $b=1$ schedules its users according to Algorithm 2, and conveys interference channels from all other BSs to its selected users. At the $i$ th step, a BS $b$, where $b=i$, selects its own users such that the channel directions of selected users have maximum spatial separation with interference channels between BS $b$ and the users already selected by BSs 1 to $b-1$. To do so, BS $b$ selects its users with maximum effective channels lying in the null space of all interference channels to the already selected users in the 


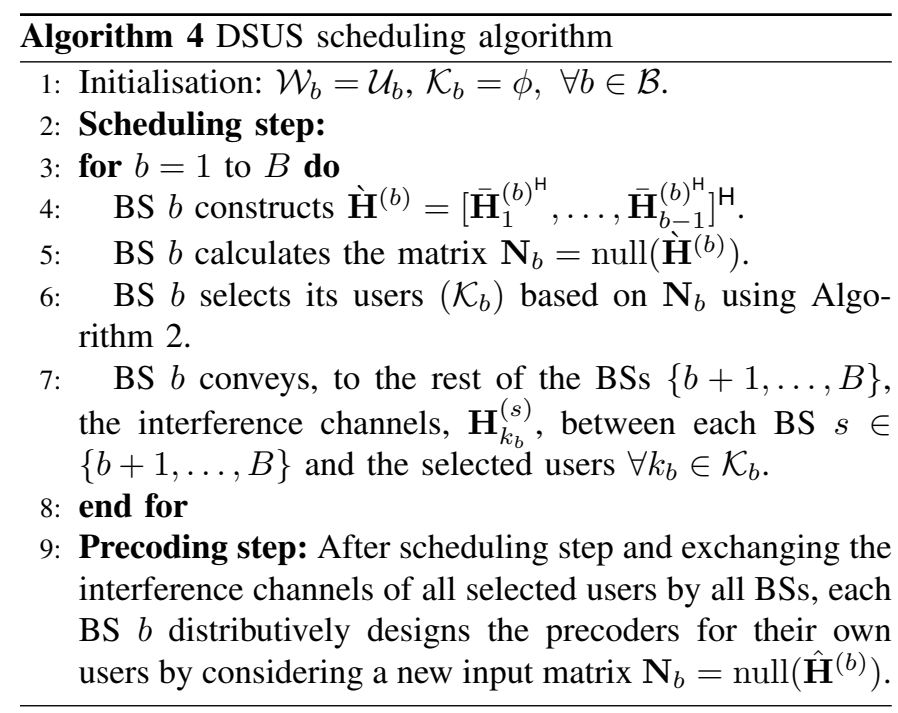

previous cells. It does so by incorporating input matrix $\mathbf{N}$, which represents the space allowed for use by its own users. To select users in each cell, an iterative algorithm determining the best users group using principal angle between subspaces is employed. Once users are selected, the BS $b$ passes the CSI of interference channels between its selected users and all other BSs. The process continues until the last BS involved in coordination selects its users. The second stage of algorithm is implemented distributively by each BS, whereby each BS, after selecting its own users, designs the transmission precoding matrices separately. The algorithm details are presented in Algorithm 4.

With this algorithm, there is no need to estimate the interference channels by all users in a cell. But only the set of selected users can estimate the CSI and feed them back to its serving BS. Thus, in addition to reducing the complexity of user selection, the algorithm can significantly reduce overhead signalling and feedback cost.

As it will be shown later, simulation results reveal that DSUS algorithm results in disparity in performance among cells. More specifically, while the first cell, i.e. BS $b=1$, obtains lower throughput compared with other cells, the last cell, i.e. BS $b=B$ enjoys best performance compared to others. This stems from the fact that the first cell in the sequence, when selecting its own users, does not take into account the interference channels to users in other cells as they are not yet selected. In contrast, the last cell knows all interference channels to the already selected users in other cells so that the $\mathrm{BS}, b=B$, can select users in a subspace which is an intersection of null spaces of all interference channels.

b) DCUS algorithm: here, the process of users selection is implemented circularly among the cells. More specifically, while in DSUS algorithm, each BS selects its set of users in one round and then the next BS does the same and the process continues until the last cell, in DCUS algorithm, each BS selects one user in its round and the next BS does the same and so on. In the next iteration, the process is repeated from

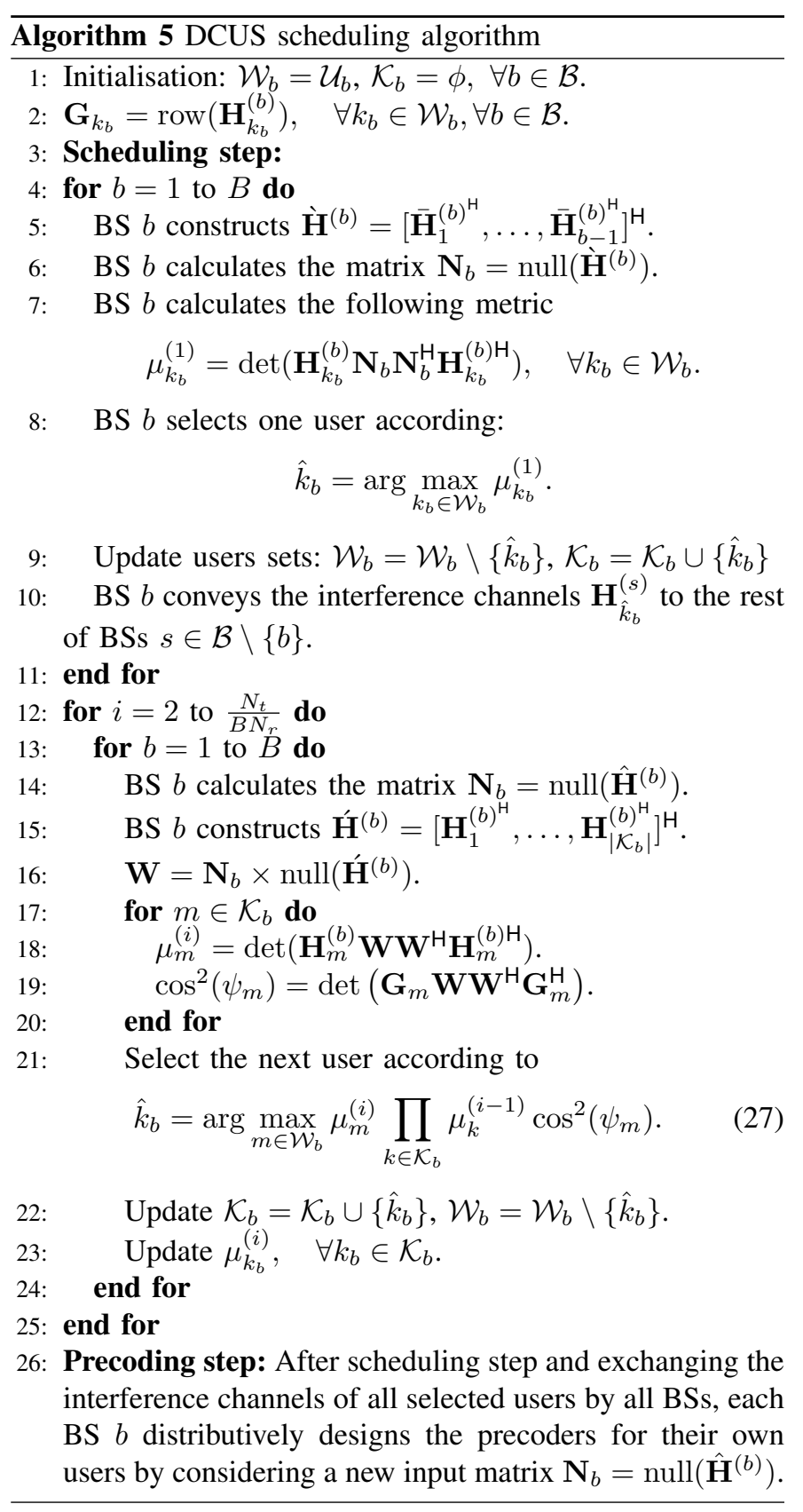

the first BS to the last one to select another single user by each cell. Thus, the process is implemented circularly among the cells. The detailed process of circular multicell scheduling is presented in Algorithm 5. The DCUS algorithm brings about more delay and frequent signalling, since each BS has to wait for other BSs to select one user per each so that it can select another single user again. However, with DCUS the disparity in sum-rates among the cells is significantly reduced, especially when users have the same average SNR, as will be evident by simulation results. Further comments will be made regarding this point in simulation results.

\section{Computational Complexity Analysis}

Here we compare the complexity of the proposed multicell user/multi-mode scheduling algorithm to that of brute-force 
search. Computational complexity is usually measured in terms of the number of flops $\varphi$ required to accomplish the whole process of calculation. A flop is defined as a real floating point operation, thus, a real addition (or multiplication) operation has one flop. While a complex addition and multiplication have two flops and six flops, respectively.

Consider a complex matrix $\mathbf{H} \in \mathbb{C}^{m \times n}$, the complexity of the following matrix operations is given as [9]

- Multiplication of an $m \times r$ complex matrix with a $r \times n$ complex matrix has $8 \mathrm{mrn}$ flops.

- Gram-Schmidt orthogonalisation (GSO) has $8 m^{2} n-2 m n$ flops.

- SVD has approximately $24 m n^{2}+48 m^{2} n+54 m^{3}$.

- $\operatorname{det}(\mathbf{H H})$ takes $8 m^{2} n+\frac{4}{3} m^{3}-\frac{3}{2} m^{2}+\frac{13}{6} m$ flops.

In the optimal multicell user/multi-mode scheduling, we assume that a central unit conducts an exhaustive search over all possible users and spatial modes combinations of which the complexity are given by (25) and (26), respectively. To find the complexity of the proposed multicell scheduling algorithm, we will consider only the first stage, i.e. user scheduling, and neglect the precoding stage. For simple notation, let $K$ denote the total number of users in each cell, i.e. $K=\left|\mathcal{U}_{b}\right|$. Then, the complexity of the proposed algorithms assuming only user selection (no multi-mode selection) can be counted for each cell as follows:

- $i=1$ : calculating both $\mu_{k_{b}}^{(1)}$ and GSO requires $K\left(16 N_{r}^{2} N_{t}-2 N_{r} N_{t}+\frac{4}{3} N_{r}^{3}-\frac{3}{2} N_{r}^{2}+\frac{13}{6} N_{r}\right)$ flops.

- $i \geq 2$ calculating $\mu_{k_{b}}^{(i)}, \cos ^{2}\left(\psi_{m}\right)$, and $\mathbf{H}_{k_{b}}^{(b)}$ needs approximately $\left(8 N_{r}^{2} N_{t}+\frac{4}{3} N_{r}^{3}-\frac{3}{2} N_{r}^{2}+\frac{13}{6} N_{r}\right), 8 N_{r} N_{t}^{2}+$ $8 N_{r}^{2} N_{t}+\frac{4}{3} N_{r}^{3}-\frac{3}{2} N_{r}^{2}+\frac{13}{6} N_{r}$, and $\left(8 N_{t}^{2} N_{r}\right)$, respectively.

For cell $b \geq 1$, it requires to calculate the null space of interference channels which takes $8(b-1)\left|\mathcal{K}_{b}\right|^{2} N_{r}$ flops. Hence, the complexity is given by

$$
\begin{aligned}
\varphi_{u s}^{\text {pro. }} & \approx \sum_{b=1}^{B}\left(K\left(16 N_{r}^{2} N_{t}-2 N_{r} N_{t}+\frac{4}{3} N_{r}^{3}-\frac{3}{2} N_{r}^{2}+\frac{13}{6} N_{r}\right)\right. \\
& +\sum_{i=2}^{\left|\mathcal{K}_{b}\right|}(K-i+1) \times\left\{8 N_{r}^{2} N_{t}+\frac{4}{3} N_{r}^{3}-\frac{3}{2} N_{r}^{2}\right. \\
& \left.\left.+\frac{13}{6} N_{r}+8 N_{t}^{2} N_{r}\right\}+8(b-1)\left|\mathcal{K}_{b}\right|^{2} N_{r}\right) \\
& \approx \mathcal{O}\left(B K\left|\mathcal{K}_{b}\right| N_{t}^{2} N_{r}\right) .
\end{aligned}
$$

The complexity ratio of the proposed algorithm to that of exhaustive search is approximately given by

$$
\xi \approx \frac{K N_{r}}{B^{2} N_{t} C\left(B K,\left\lceil B N_{t} / N_{r}\right\rceil\right)},
$$

which shows dramatic reduction in computational complexity for multicell scheduling.

\section{Proportional Fair Scheduling}

While Algorithms 4 and 5 described in Section III aim at maximising system throughput, they will always favour users with better channel conditions. Under practical situations, when users may stay at cell-edge for a long time, this gives rise to fairness issues, i.e. cell-edge users may not get scheduled for long time as long as they stay on cell boundaries. Fair scheduling is proposed to guarantee a certain degree of fairness among the users in the system. Various fair scheduling policies are proposed in literature, however in this work we consider proportional fairness scheduler. To keep the limited space, we consider multi-mode selection with proportional fairness (PF) scheduler problem, which can be defined as

$$
R^{P F}=\arg \max _{\mathcal{K}_{b} \in \mathcal{U}_{b}, L_{k_{b}}, \mathbf{P}_{k_{b}}, \mathbf{Q}_{k_{b}}} \sum_{b=1}^{B} \sum_{k_{b} \in \mathcal{K}_{b}} \frac{R_{k_{b}}(t)}{\bar{R}_{k_{b}}(t)},
$$

with the same constraints in $(20-24)$. In this problem, $R_{k_{b}}(t)$ is the rate of the user $k_{b}$ at time instant $t$. While $\bar{R}_{k_{b}}(t)$ is the average rate of user $k_{b}$. When setting $\bar{R}_{k_{b}}(t)$ to 1 the problem is reduced to that of (19), which is identical to maximising the sum-rate. In PF, $\bar{R}_{k_{b}}(t)$ is updated as

$$
\bar{R}_{k_{b}}(t+1)= \begin{cases}\left(1-\frac{1}{t_{c}}\right) \bar{R}_{k_{b}}(t)+\frac{1}{t_{c}} R_{k_{b}}(t), & \text { if selected } \\ \left(1-\frac{1}{t_{c}}\right) \bar{R}_{k_{b}}(t), & \text { if not selected }\end{cases}
$$

where $t_{c}$ is window time. The PF scheduler ensures the fairness by giving priority to the users with sufficiently large value of $\frac{R_{k_{b}}(t)}{\bar{R}_{k_{b}}(t)}$. Thus, users with low average rate $\bar{R}_{k_{b}}(t)$ are more likely to be served in the subsequent scheduling intervals. The problem of PF with user selection lies in the fact that the exact rate $R_{k_{b}}$ of user $k_{b}$ is unknown before completion of user selection process. However, this problem can be overcome by assuming constant power allocation, which is near optimal in high SNR regime. Let $L_{k_{b}}^{i}$ be the number of spatial modes at $i$ th iteration for the user $k_{b}$ including the stream to be scheduled next, denoted as $\hat{l}$. The rate of user $k_{b}$ in the $i$ th iteration step can be approximated as:

$$
R_{k_{b}}^{i} \approx \sum_{l=1}^{L_{k_{b}}} \log _{2}\left(1+\frac{P}{i L_{k_{b}}^{i}} g_{k_{b}}^{i}\right)
$$

where

$$
g_{k_{b}}^{i}=\prod_{l \in \mathcal{S}_{k_{b}} \cup\{\hat{l}\}} \mu_{k_{b}, l}^{i}
$$

where $g_{k_{b}}^{i}$ represents the product of squared row norms of the effective channels. The above metric can be further simplified assuming high SNR regime as

$$
R_{k_{b}}^{i} \approx \log _{2}\left(\left(\frac{P}{i L_{k_{b}}^{i}}\right)^{L_{k_{b}}^{i}} g_{k_{b}}^{i}\right)
$$

The multi-mode selection metric for PF scheduler at the $i$ th iteration is replaced by:

$$
\left(\hat{k}_{b}, \hat{l}\right)=\arg \max _{m \in \mathcal{W}_{b} ; l \in \mathcal{A}_{k_{b}}} \frac{R_{k_{b}}^{i}}{\bar{R}_{k_{b}}}+\sum_{k \in \mathcal{Z}_{b}} \sum_{j \in \mathcal{S}_{k_{b}}} \frac{R_{k_{b}}^{i}}{\bar{R}_{k_{b}}} .
$$

To consider PF in users/modes selection, (35) replaces the metric in step (14) in Algorithm 3. 


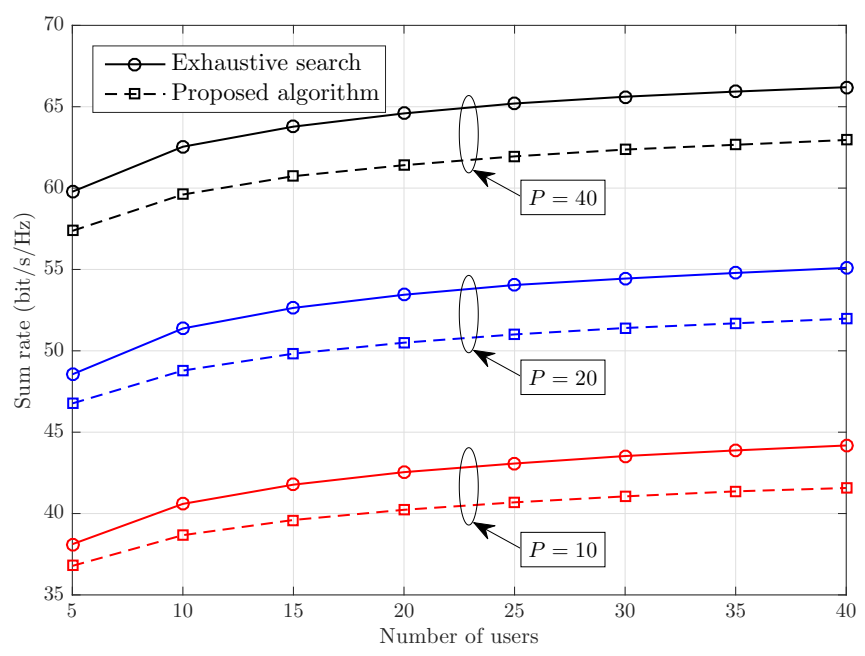

Fig. 2. Comparison between an optimal solution obtained by exhaustive search and the proposed algorithm for different power values.

\section{Simulation Results}

We consider a multicell system consisting of three hexagonal cells $(B=3)$ with radius of $R=1 \mathrm{~km}$. Each $\mathrm{BS}$ is equipped with $N_{t}=12$ and employs BD while coordinating toward users served by other cells. Users, each equipped with two antennas $\left(N_{r}=2\right)$, are randomly and uniformly distributed within the cell area. The users are assumed to always have data for transmission (full buffer assumption). Although the proposed scheme can accommodate multicarrier systems, we only consider single subchannel with bandwidth of $180 \mathrm{kHz}$. The channel is assumed to be subjected to three components; path-loss, lognormal shadowing, and small-scale Rayleigh fading. The path-loss model is given by:

$$
\hat{\beta}_{k_{b}}^{i}(\mathrm{~dB})=136+40 \log _{10}\left(d_{k_{b}}^{i}\right),
$$

where $d_{k_{b}}^{i}$ is the distance given in $\mathrm{km}$ between the user $k_{b}$ and a BS $i$. Noise spectral density of $-174 \mathrm{dBm} / \mathrm{Hz}$ and $8 \mathrm{~dB}$ standard deviation for Shadowing are considered.

Fig. 2 depicts the total sum-rate in bit $/ \mathrm{s} / \mathrm{Hz}$ versus the total number of users for various power $(P)$ values, averaged over $10^{5}$ channel realisations. The figure shows the performance of exhaustive search and the proposed algorithm DSUS with fixed spatial mode (conventional BD). It can be clearly observed that the proposed algorithm achieves most of the sum-rate achievable by exhaustive search. It can be also noticed that the sum-rate is increased with the increase of total number of users, which is due to multi-user diversity.

Fig. 3 shows a comparison between DSUS and DCUS algorithms assuming user pairing in which users are assumed to have the same average signal-to-noise ratio (SNR) so that only small-scale fading is taken into account. As expected, we figure out that the gap in performance among cells in sequential algorithm (DSUS) is larger than that of circular algorithm (DCUS). This comes in an agreement with our expectation that the order in which the process of multicell scheduling is accomplished allows the latest cells to select users whose channels directions lie almost in the null space of interference channels of all other users belonging to other cells. In contrast,

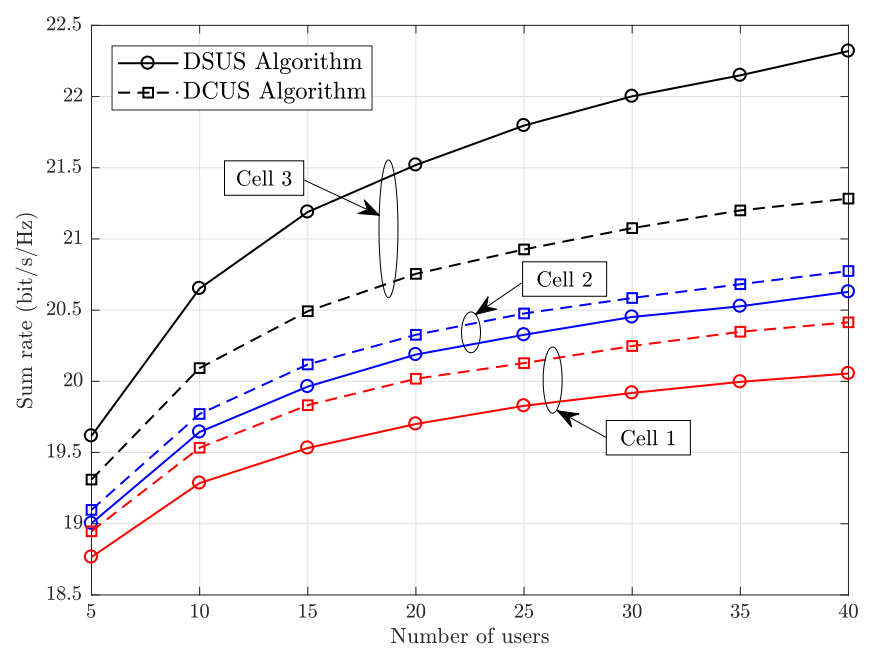

Fig. 3. Comparison between rates of the cells obtained by the two proposed algorithms DSUS and DCUS $(P=40)$.

the previous cells in the sequence of algorithm have no knowledge at their turn about other next cells decision regarding user selection; consequently they may select users whose channels directions may not lie in the null space of interference channels to other cells users. Furthermore, we expect that the gap increases with the increase in the number of cells involved in coordination accordingly. On the other hand, in DCUS, since one user is selected by each cell in its turn, this allows the first cells in sequence to have knowledge about more interference channels to other cells users, while making the latest cells have knowledge about fewer interference channels, hence yielding more fairness among the cells in terms of sum-rate. However, when the users suffer heterogeneous channel conditions, the gap tends to diminish and then both algorithms will have comparable performance due to higher multi-user diversity gain provided by heterogeneity of users channels. The fairness issue may disappear in the long term for the DSUS algorithm by changing the order of BSs. However, for a given iteration, the first BS will have better performance compared to the last BS. On the other hand, in DCUS algorithm, since each BS selects only one user each time, the fairness among the BSs can be enhanced by rotating the list of BSs. For example, let us suppose that we start with BS 1 , until BS $n$, now if we start again from $\mathrm{BS} n$, until $\mathrm{BS} 1$, we expect that the gap between BSs becomes ever smaller with the increasing number of users selected per each cell.

Fig. 4 compares between fixed spatial mode (conventional BD) and multi-mode selection with the DSUS algorithm in terms of sum-rate for versus number of users. As can be clearly observed, multi-mode selection outperforms fixed spatial mode scheme with only user selection. The gain comes from the flexibility offered by multi-mode selection to choose the best set of channel direction on the level of antennas, thereby exploiting the spatial diversity more efficiently.

In Figs. 5 and 6, compare between sum-rate maximization (SRM) and PF scheduling schemes. The total rate of the system and the sum-rate of cells are depicted versus number of users, respectively. The observed decrease in sum-rate in PF as 


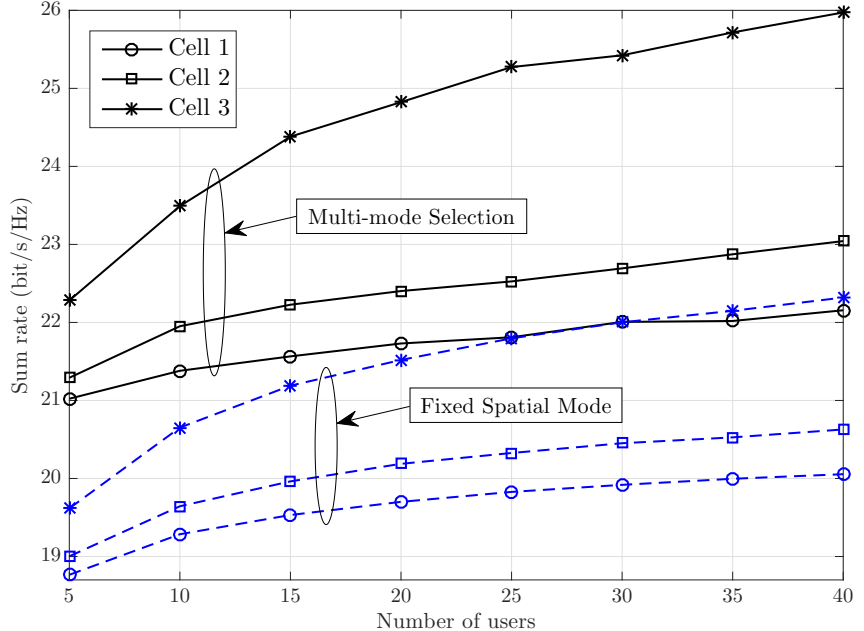

Fig. 4. Comparison between rates of the cells obtained by conventional BD and multi-mode selection scheme $(P=40)$.

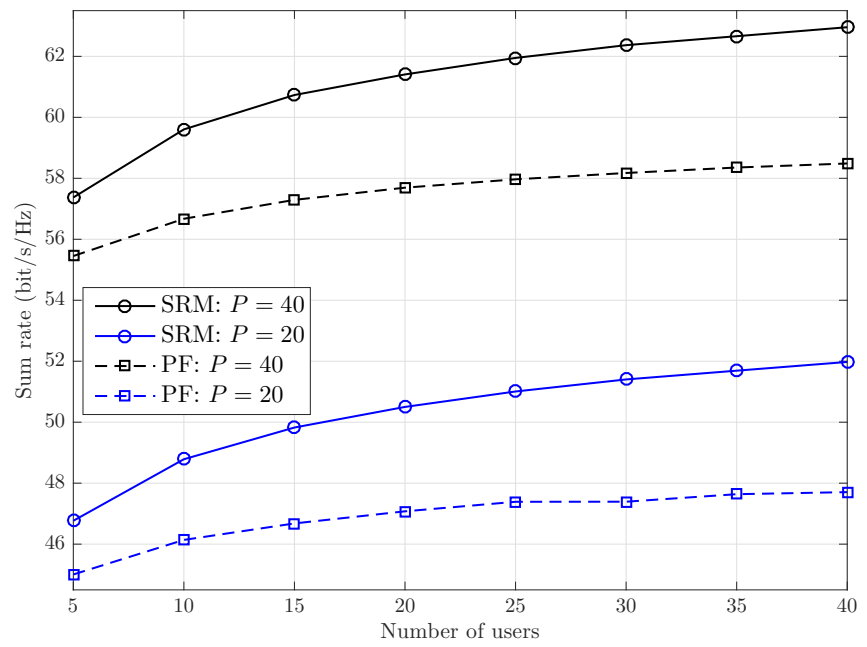

Fig. 5. The figure compares between SRM and PF scheduling schemes in terms of sum of rates of the cells in the system versus number of users.

compared with SRM comes from the fact that PF attempts to guarantee fairness as described previously. However, PF will ensure that users with bad channel conditions to be served.

\section{CONCLUSIONS}

In this paper, multicell scheduling for coordinated MIMO cellular network is investigated and analysed. Two low complexity algorithms are proposed for user scheduling with conventional $\mathrm{BD}$ and multi-mode selection. The algorithms are based on two stages: multicell scheduling and precoding stages, and work in progressive sequential manner. Thus, lower computation complexity and overhead signalling are required for algorithms operation. While the distributed sequential user scheduling algorithm is shown to give higher priority for the latest cells in the sequence order, the distributed circular user scheduling achieves better fairness among cells. Furthermore, the algorithms are extended to accommodate proportional fairness scheduling. Simulation results show high percentage

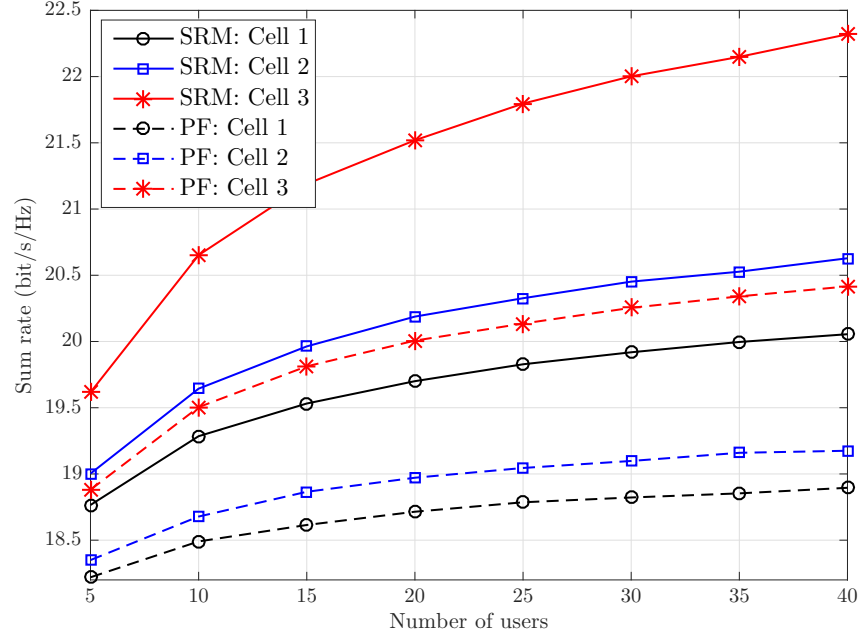

Fig. 6. Comparison between SRM and PF scheduling schemes in terms of sum rates of individual cells $(P=40)$.

of sum-rate achievable via exhaustive search can be achieved by these algorithms.

\section{REFERENCES}

[1] Cisco, "Cisco visual networking index: global mobile data traffic forecast update, 20132018," White Paper, Feb. 2014. [Online]. Available: http://www.cisco.com.

[2] Cisco, "3GPP LTE Release 10 \& Beyond (LTE-Advanced), 3rd Generation Partnership Project (3GPP)," 2009. [Online]. Available: http://www.3gpp.org/.

[3] A. S. Hamza, S. S. Khalifa, H. S. Hamza, and K. Elsayed, "A survey on inter-cell interference coordination techniques in OFDMA-based cellular networks," IEEE Communications Surveys \& Tutorials, vol. 15, no. 4, pp. 1642-1670, 2013.

[4] J. Mietzner, R. Schober, L. Lampe, W. H. Gerstacker, and P. A. Hoeher, "Multiple-antenna techniques for wireless communications a comprehensive literature survey," IEEE Communications Surveys \& Tutorials, vol. 11, no. 2, pp. 87-105, 2009.

[5] H. Weingarten, Y. Steinberg, and S. S. Shamai, "The capacity region of the Gaussian multiple-input multiple-output broadcast channel," IEEE Transactions on Information Theory, vol. 52, no. 9, pp. 3936-3964, Sep. 2006.

[6] T. Yoo and A. Goldsmith, "On the optimality of multiantenna broadcast scheduling using zero-forcing beamforming," IEEE Journal on Selected Areas in Communications, vol. 24, no. 3, pp. 528-541, Mar. 2006.

[7] Q. H. Spencer, A. L. Swindlehurst, and M. Haardt, "Zero-forcing methods for downlink spatial multiplexing in multiuser MIMO channels," IEEE Transactions on Signal Processing, vol. 52, no. 2, pp. 461-471, Feb. 2004.

[8] G. Dimic and N. D. Sidiropoulos, "On downlink beamforming with greedy user selection: performance analysis and a simple new algorithm," IEEE Transactions on Signal Processing, vol. 53, no. 10, pp. 3857-3868, Oct. 2005

[9] Z. Shen, R. Chen, J. G. Andrews, R. W. Heath, and B. L. Evans, "Low complexity user selection algorithms for multiuser MIMO systems with block diagonalization," IEEE Transactions on Signal Processing, vol. 54, no. 9, pp. 3658-3663, Sep. 2006.

[10] L. N. Tran, M. Bengtsson, and B. Ottersten, "Iterative precoder design and user scheduling for block-diagonalized systems," IEEE Transactions on Signal Processing, vol. 60, no. 7, pp. 3726-3739, July 2012.

[11] T. F. Maciel and A. Klein, "On the performance, complexity, and fairness of suboptimal resource allocation for multiuser MIMO-OFDMA systems," IEEE Transactions on Vehicular Technology, vol. 59, no. 1, pp. 406-419, Jan. 2010.

[12] X. Yi and E. K. S. Au, "User scheduling for heterogeneous multiuser MIMO systems: A subspace viewpoint," IEEE Transactions on Vehicular Technology, vol. 60, no. 8, pp. 4004-4013, Oct. 2011.

[13] S. Nam, J. Kim, and Y. Han, "A user selection algorithm using angle between subspaces for downlink MU-MIMO systems," IEEE Transactions on Communications, vol. 62, no. 2, pp. 616-624, Feb. 2014. 
[14] S. Sigdel and W. A. Krzymie, "Simplified fair scheduling and antenna selection algorithms for multiuser MIMO orthogonal space-division multiplexing downlink," IEEE Transactions on Vehicular Technology, vol. 58, no. 3, pp. 1329-1344, Mar. 2009.

[15] R. Chen, Z. Shen, J. G. Andrews, and R. W. Heath, "Multimode transmission for multiuser MIMO systems with block diagonalization," IEEE Transactions on Signal Processing, vol. 56, no. 7, pp. 3294-3302, July 2008.

[16] D. Gesbert, S. Hanly, H. Huang, S. S. Shitz, O. Simeone, and W. Yu, "Multi-cell MIMO cooperative networks: A new look at interference," IEEE Journal on Selected Areas in Communications, vol. 28, no. 9, pp. 1380-1408, Dec. 2010.

[17] H. Dahrouj and W. Yu, "Coordinated beamforming for the multicell multi-antenna wireless system," IEEE Transactions on Wireless Communications, vol. 9, no. 5, pp. 1748-1759, May 2010.

[18] L. Venturino, N. Prasad, and X. Wang, "Coordinated linear beamforming in downlink multi-cell wireless networks," IEEE Transactions on Wireless Communications, vol. 9, no. 4, pp. 1451-1461, Apr. 2010.

[19] Y. F. Liu, Y. H. Dai, and Z. Q. Luo, "Coordinated beamforming for MISO interference channel: Complexity analysis and efficient algorithms," IEEE Transactions on Signal Processing, vol. 59, no. 3, pp. 1142-1157, Mar. 2011

[20] J. Zhang, R. Chen, J. G. Andrews, A. Ghosh, and R. W. Heath, "Networked MIMO with clustered linear precoding," IEEE Transactions on Wireless Communications, vol. 8, no. 4, pp. 1910-1921, Apr. 2009.

[21] D. Choi, D. Lee, and J. H. Lee, "Resource allocation for CoMP with multiuser MIMO-OFDMA," IEEE Transactions on Vehicular Technology, vol. 60, no. 9, pp. 4626-4632, Nov. 2011.

[22] S. Kaviani and W. A. Krzymien, "Multicell scheduling in network MIMO," in IEEE Global Telecommunications Conference, Dec 2010, pp. 1-5.

[23] G. H. Golub and C. F. V. Loan, Matrix Computations, 3rd ed. Johns Hopkins University Press, 1996.

[24] O. N. H. Gunawan and W. Setya-Budhi, "A formula for angles between two subspaces of inner product spaces," Beiträge zur Algebra und Geometrie, vol. 46, no. 2, pp. 311-320, 2005.

[25] A. Björck and G. H. Golub, "Numerical methods for computing angles between linear subspaces," Mathematics of Computation, vol. 27, no. 123 , pp. 579-594, July 1973.

[26] I. B. Risteski and K. G. Trenčevski, "Principal values and principal subspaces of two subspaces of vector spaces with inner product," Beiträge zur Algebra und Geometrie, vol. 42, no. 1, pp. 289-300, 2001. 